\title{
Competitive Evolution of Tactical Multiswarm Dynamics
}

\author{
Albert R. Yu, Benjamin B. Thompson, and Robert J. Marks, II, Fellow, IEEE
}

\begin{abstract}
The dynamics of large decentralized groups of agents, or swarms, can be difficult to characterize due to complex and often unpredictable behaviors that arise from low-level interactions between agents. When designing multiagent systems, these emergent behaviors can have hidden and undesirable implications on the overall operation of the swarm. This paper examines the use of inversion of swarm dynamics to refine individual agents' rules of operation in order to achieve a given collective goal and applies this method to a scenario of tactical relevance: the point defense of a very important person between two attacking and defending swarms. An alternating competitive evolution is used in a toggled behavioral arms race in order to refine tactics and anticipate counteractions. Results include creative solutions with varying levels of success at addressing defensive tactical scenarios, with the attacking swarms evolving behaviors (such as rushing, splitting, and baiting) and the defending swarm evolving proactive and reactive solutions.
\end{abstract}

Index Terms-Competitive evolution, genetic algorithm, multiagent systems, swarm intelligence.

\section{INTRODUCTION}

A UTONOMOUS multiagent system dynamics can be difficult to determine from the low-level interactions of individual agents. How complex systems develop from undirected local relationships is often obscure and is the focus of swarm intelligence research. Identifying the emergent properties and behaviors of a swarm, given individual agent-operating parameters, can be difficult without simulation or implementation [3], [9], and training agents to accomplish a given task grows in complexity with larger groups due to the plurality of interactions. Hand-tuning the operating scheme of a complex system to perform over a prolonged period of time can generate unintended and counterintuitive emergent behaviors, and largescale deterministic plans may fail if the operational theater is perturbed.

Manuscript received August 12, 2011; revised January 17, 2012; accepted May 31, 2012. Date of publication February 1, 2013; date of current version April 12, 2013. This work was supported by the U.S. Office of Naval Research University-Laboratory Initiative N000140910398 and N000140910434. This paper was recommended by Associate Editor W. Pedrycz.

A. R. Yu is with the University of Washington, Seattle, WA 98195 USA (e-mail: Albert_Yu@baylor.edu).

B. B. Thompson is with Pennsylvania State University, University Park, PA 16802 USA (e-mail: bbt10@psu.edu).

R. J. Marks, II is with the Department of Electrical and Computer Engineering, Baylor University, Waco, TX 76798 USA (e-mail: Robert_Marks@ baylor.edu)

Color versions of one or more of the figures in this paper are available online at http://ieeexplore.ieee.org.

Digital Object Identifier 10.1109/TSMCA.2012.2210410
Modeling competition is rarely performed at the level of individual agent dynamics due to computational constraints and the elusiveness of an inclusive accurate model. Competitive population dynamics, such as predator-prey interactions, are typically generalized at a high level. Models such as the classical Lotka-Volterra [12], [18] examine overarching static population dynamics, including indefinite factors such as birth and mortality rates as an approximation for the low-level relationships between individual agents. Alternatively, swarm theory can provide a framework for investigating the tactical responses of agents within a population through inversion of swarm dynamics [8]. This paper examines the efficacy of genomic inversion in developing the behavioral dynamics of tactical relevance in a multiswarm competitive predator-prey scenario and specifically applies it to a variant of the weapontarget assignment problem (WTA) [13].

WTA problems are a historically well-studied field of optimization. In single-weapon-type WTA problems, a given number of weapons must efficiently allocate targets in order to maximize some objective function, such as to maximize damage, probability-to-hit, or kill ratios. Solutions to WTA problems include the use of linear programming, negotiation techniques [17], and neural network models [19]. However, these scenarios are often based on situations where global or direct local communication is possible, or high-level target allocation is determined a priori and the dynamics of weapons are simplified to hit probabilities. In environments where such communication is not possible, the system can be characterized by the emergent behavior of indirect local interactions through swarm theory.

The emergent behavioral response of two competing similarly-sized swarms can be examined through simulation. Swarm inversion is an improvement algorithm that is meant to refine the solution toward an improved but not necessarily optimal solution. Emergent behavior is an aspect of swarm intelligence and refers to large groups of agents interacting under simple rules that exhibit some added cooperative benefit and has applications in communications [1], [5], [11], robotics [2], [3], [6], and optimization [4], [13], [14], [20]. Swarms often demonstrate robustness, plasticity, and decentralization [4], which are ideal characteristics for modeling autonomous large-group interactions.

The goal of this paper is to outline a simulation-based evolutionary method of determining the optimal rules of operation for competing swarms in accomplishing some objective. Evolving decision rules and plans via simulation to determine a "tactical plan" has been done before; Grefenstette et al., applied a similar method for evolving a rulebook encompassing all 
decisions in a given sequential decision task in an aerial evasion problem [10]. Often, the goal of behavioral optimization is to produce theoretical genotypes that are capable of defeating all opponents. However, this notion of a universal behavioral tactic does not apply to most complex games. Instead, a choice of an optimal set of controller behaviors or playbook that divulges an appropriate response depending on the nature of an opponent is preferred. The objective is to use evolutionary techniques to find not pervading dominant tactics but a viable natural progression of tactical responses in a given situation. This is achieved using disjunctive Combs control, as presented by Ewert et al. [7]. In contrast with other inversion approaches [7], each swarm agent's sensor readings are altered by a nonlinear actuator whose shape is determined by the inversion process. Actuator outputs are then aggregated to determine the agent's actions within the swarm.

\section{Methodology}

\section{A. Scenario Description}

The scenario of interest is the swarming defense of a very important person (VIP) from being caught by a swarm of equal size to the defending swarm. Both attacker and defender swarms are initialized randomly within fixed proximities from the VIP. Attackers initialize randomly on a semicircular arc at the edge of the theater, whereas defenders initialize near the VIP. The inspiration for this scenario is the defense of a relatively immobile target against large-scale suicide attacks, (e.g., suicide bombers or guided missiles.) This is an objectivebased extension of the classic predator-prey model; the attacking swarm preys on the VIP, and the defending swarm pursues the attackers. All agents have the capability of disabling each other, but only defenders are rewarded for doing so. Agents attack by detonating and disabling any friend or foe within a fixed blast radius. Attacker fitness is based on the number of hits against the objective. A time or fuel limit is imposed to address stalemates, which reward in favor of the defenders.

Agent autonomy is a primary concern in designing both swarms. The attacker and defender swarms are homogeneous with limited interactive abilities and no specific role assignment mechanisms. Homogeneity is enforced to prevent overspecialization and to direct the evolution of the swarm into developing a strong base set of rules of operation. The capabilities of each side are assumed to be equal; all agents utilize the same maximum speed, update frequency, and maximum sensing range. Each agent is aware of its global position, as well as all other agents within a fixed range. However, there is no direct communication; agents can infer position and velocity from nearby allies but not visual or target information. In this scenario, agents reliably discern friend from foe, noting the closest ally, but not his identity, as well as the most threatening enemy.

The threat of an enemy reflects the team's objectives. For attackers, the designated threat of a defender is proximity, as defenders are rewarded for attacker kills. For defenders, the threat of an attacker is based on whether that target has been handled by the rest of the swarm. Since the number of attackers

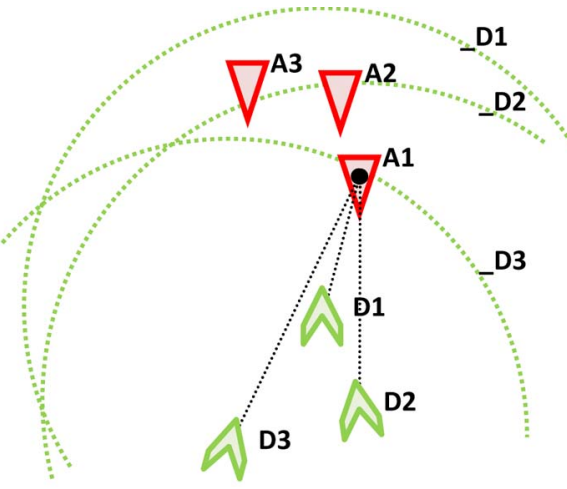

(a)

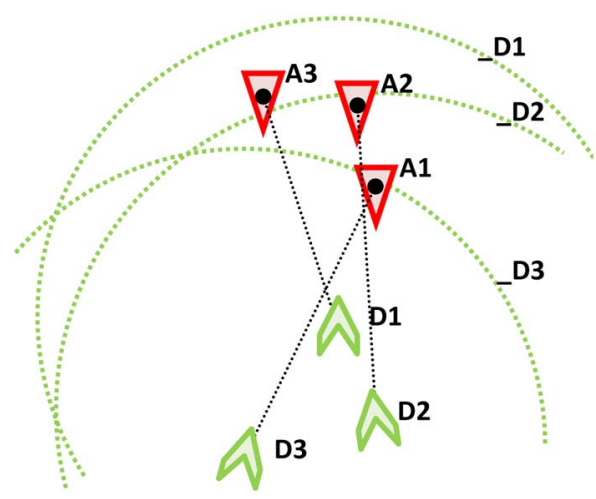

(b)

Fig. 1. In (a), defenders (chevrons) select attacker (triangles) targets based on proximity. This results in the defenders all choosing Target A to intercept. In (b), defenders evaluate targets based on their perceptions. Defenders D1 and D2 recognize that D3 can only see Attacker A1, so they assume A1 is covered by $\mathrm{D} 3$. Of the remaining targets, A2 is the closest to both defenders. To minimize the total travel distance to targets for both agents, D1 selects the farther A3 and assumes that D2 will select A2. If D2 perceives the same world as D1, then it will make the same assignment. This scheme defaults to the closest target when the number of local defenders exceeds attackers. Allocation scheme (b) was found to be sufficient despite the each agent's limited sensing. (a) Target assignment via closest target. (b) Target assignment via minimum group distance under visibility constraints.
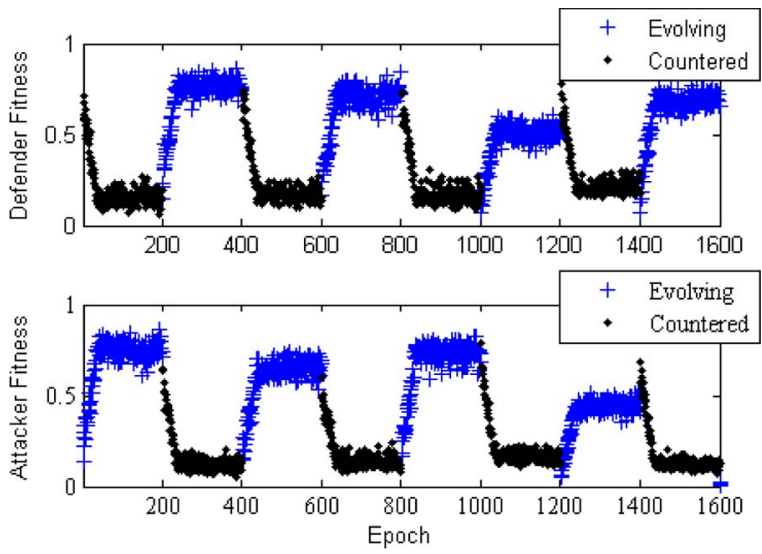

Fig. 2. Defender and attacker fitness per iteration. Attackers evolve for the first 200 iterations, and then, defenders counterevolve for 200 iterations. This cycle is repeated. While the performance of each team population appears cyclical and typical of the evolution, the actual behaviors that emerge are not the same.

and defenders is equal and there is no direct communication between any agents, defenders cannot know if each defender has been assigned the best attacker to neutralize. As agents move in and out of visual range, each agent is potentially 

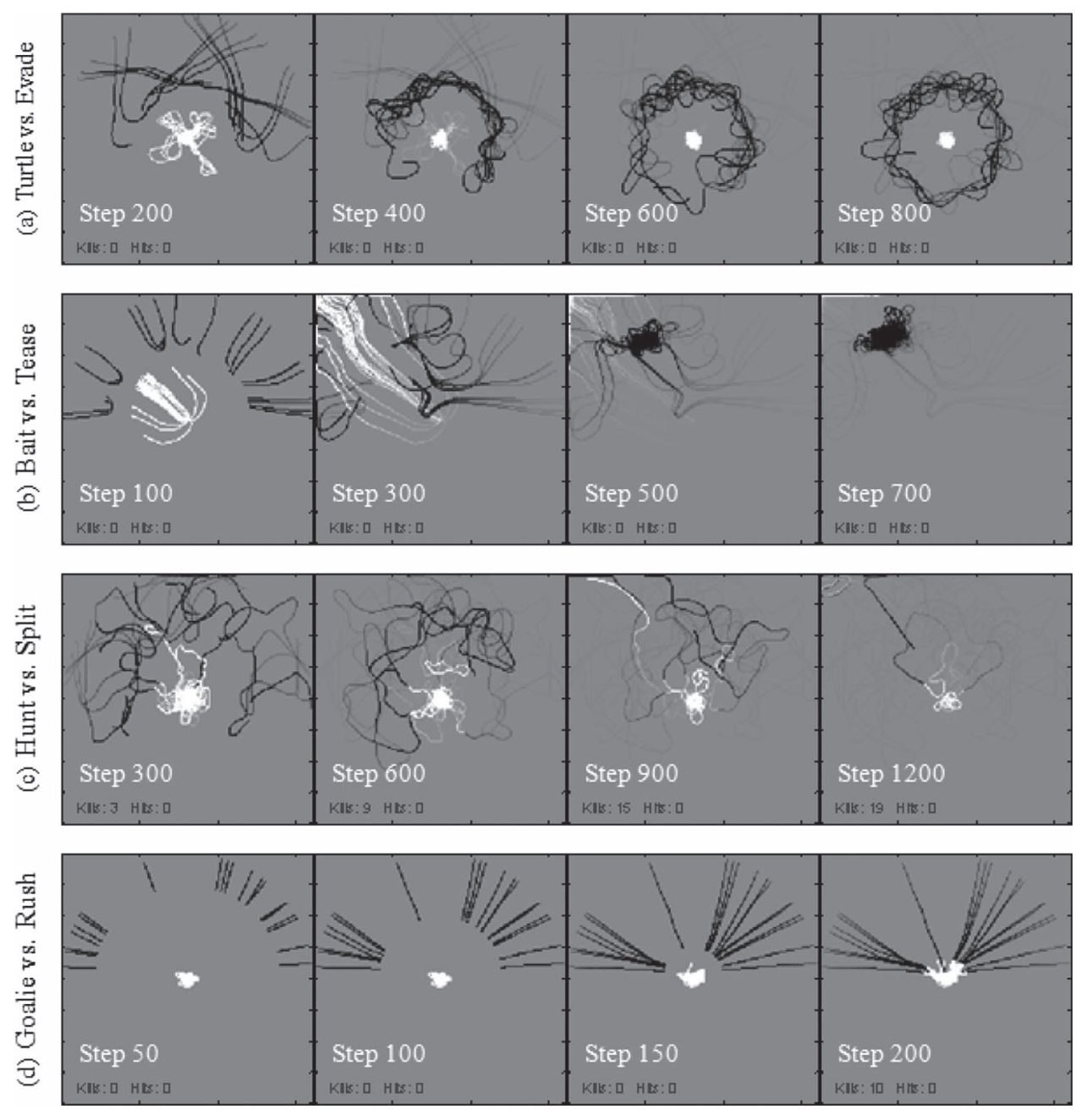

Fig. 3. Defender (white trails) counters to attacker (black trails) behaviors. (a) Defenders tightly cluster around the VIP, exploiting the attackers' reluctance to engage. (b) Defenders take advantage of the attackers' teasing attachment, a behavior used by attackers to draw defenders into wild chases, by leading the attackers away from the VIP and running out the clock. (c) Defenders leave the center to chase down targets. (d) Defenders await to intercept rushing attackers where attacker movement is most constricted.

introduced to new target and ally information. Simply selecting the closest target is often suboptimal when allies are involved; another ally may be the better interceptor for the closest target despite being farther away. Instead, a minimized cost algorithm based on the shortest-path distance is implemented for threat analysis and target allocation in the neighborhood defined by each agent's sensor range (Fig. 1).

\section{B. Parameterization}

A nine-element array is used to represent a team's genome. These genomes represent the swarm's behavioral response to a given sensor. Agents have sensors that determine the agent's range from the VIP, as well as that of all allies and enemies within its visual range. After assessing enemy threats, agents respond to three antecedents: the relative position of the 1) highvalue target, 2) closest ally, and 3) greatest enemy threat within visual range. The range to each of these antecedents is passed through an evolved piecewise-linear response defined by three evolved parameters each. The resulting three consequents are summed to form the final response vector for the agent, subject to morphological constraints.

\section{Evolution, Counterevolution, and Fitness}

The goal of the evolutionary process is to identify behaviors that can be used to defeat an opposing swarm. However, the tactics employed by the opposing force may not always be the same, and optimizing a swarm against one possible attack or defense leaves the swarm vulnerable to being countered by a different behavior. There is rarely a universal tactic for all situations; instead, a playbook of potential tactics for the corresponding forcing conditions is desirable. This is inspired by coevolution's asymmetrical evolutionary arms race; however, behavioral responses are developed sequentially, instead of concurrently. One side makes a behavioral breakthrough and exploits that solution until the other team discovers its own countersolution. The fitness in each case is a function of the solution of the result of the evolution of the opponent and thus dynamically changes.

The evolutionary technique applied is a modified genetic algorithm. The attacking team is evolved against the best defender genome. Then, the defending team is optimized against the resulting best attacker genome, and the cycle is repeated. Mutation, crossover, and elitism occur, and all past high performers are used to repopulate each new cycle. In addition, 

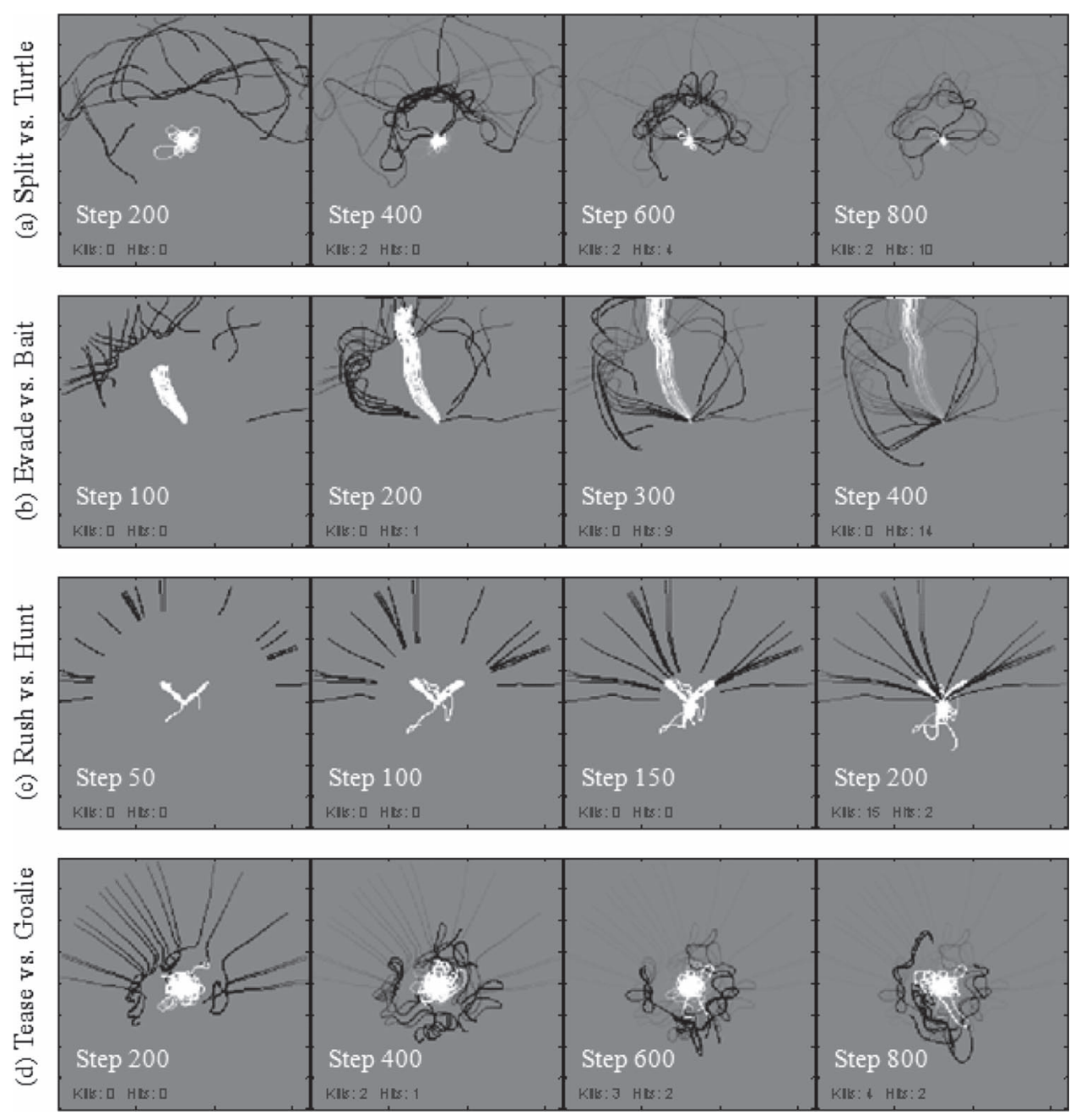

Fig. 4. (Black trails) Attacker counters to (white trails) defender behaviors. (a) Attackers disperse, leaving one attacker to attack at a time to break a defender bunker and lowering the VIP's defense. (b) Attackers are repelled by defenders at any distance, letting them avoid any casualties and overcome baits and feints. (c) Rushing attackers ignore defenders completely, falling through the cracks as defenders leave the center to intercept them. (d) Attackers lead defenders on wide chases, eventually slipping through cracks in the defense.

a variable number of genomes are reinitialized during each iteration to help maintain population diversity. The result of five runs are averaged to determine the matchup's fitness for both swarms as there is a random component to both the initialization of the swarms in the simulator and a jitter in their movements. For attackers, fitness is based on their hit ratio, relative to the total number of attackers. For defenders, fitness is the average win rate of the genome out of those five games.

To initiate this cycle, each team's genome is generated randomly. Opposing genomes are paired, and the highest performing genome for the attackers becomes the first round opponent for the defenders. This procedure serves as a random starting point for the evolutionary race. Defenders are evolved against this attacker until the optimal defender is determined. The focus then shifts to attackers, evolving their behavior until a suitable performance level is achieved. These cycles are repeated, and the final behaviors on both sides are compared.

\section{Simulator}

A MATLAB script was written to perform the simulation and invert the swarms. Swarms of 20 attackers and 20 defenders were used. Agents had 1200 time steps in order to complete an attack.

\section{Results}

\section{A. Fitness}

The results of the simulation's cyclical evolutions are shown in Fig. 2. A population size of 50 genomes is used for both attackers and defenders. A limit of 200 generations is chosen due to the genetic algorithm's propensity to quickly converge. When a team is evolved, there is an observed spike in the performance of the opponent as the population reinitializes, but this effect is quickly nullified.

\section{B. Qualitative Observations}

In general, both attackers and defenders would cyclically evolve similar sets of behaviors when developing counters against each other. Qualitative observations were used for a naming scheme of the behaviors that arose, as observed in Figs. 3 and 4. Four repeatedly observed behaviors for defenders and attackers are listed in Tables 1 and 2, respectively. The 
TABLE I

DEFENDER BEHAVIORS

\begin{tabular}{|c|c|c|c|}
\hline Name & Primary Rules & Description & Counters \\
\hline (a) Turtle & $\begin{array}{l}\text { Stay near VIP } \\
\text { Ignore Enemies }\end{array}$ & $\begin{array}{l}\text { Defenders hide in the center repel attackers } \\
\text { unwilling to self-sacrifice. }\end{array}$ & $\begin{array}{l}\text { Evader } \\
\text { Teaser }\end{array}$ \\
\hline (b) Bait & $\begin{array}{l}\text { Avoid VIP } \\
\text { Stick Together }\end{array}$ & $\begin{array}{l}\text { Defenders exploit the attacker's pulsing or teasing } \\
\text { behavior by drawing them out and away from the } \\
\text { VIP }\end{array}$ & Teaser \\
\hline (c) Hunter & Pursue Enemies & Defenders actively pursue targets when provoked. & Split \\
\hline (d) Goalie & $\begin{array}{l}\text { Stay near VIP } \\
\text { Pursue Enemies }\end{array}$ & $\begin{array}{l}\text { Defenders swarm near the VIP, intercepting } \\
\text { targets but rarely leaving the VIP's immediate } \\
\text { proximity. }\end{array}$ & Rusher \\
\hline
\end{tabular}

TABLE II

ATTACKER BEHAVIORS

\begin{tabular}{|c|c|c|c|}
\hline Name & Primary Rules & Description & Counters \\
\hline (a) Splitters & Avoid allies & $\begin{array}{l}\text { Disperses the attackers and subsequently any pursuers. } \\
\text { Against dense defenses, slips in one attacker at a time. }\end{array}$ & $\begin{array}{l}\text { Turtle } \\
\text { Bait }\end{array}$ \\
\hline (b) Evaders & Avoid enemies & Avoids defenders, making baits particularly ineffective. & Bait \\
\hline (c) Rushers & $\begin{array}{l}\text { Ignore enemies } \\
\text { Pursue VIP }\end{array}$ & $\begin{array}{l}\text { Ignore defenses for a quick win. Effective when } \\
\text { defenders pick optimum targets but cannot reposition } \\
\text { themselves in time, or against dense clusters of enemies } \\
\text { as individual blasts disproportionately disable defenders. }\end{array}$ & $\begin{array}{l}\text { Turtle } \\
\text { Hunter } \\
\text { Bait }\end{array}$ \\
\hline (d) Teasers & Pulse enemies & $\begin{array}{l}\text { Attackers pulse defenders, drawing them out and opening } \\
\text { cracks. }\end{array}$ & $\begin{array}{l}\text { Goalie } \\
\text { Hunter }\end{array}$ \\
\hline
\end{tabular}
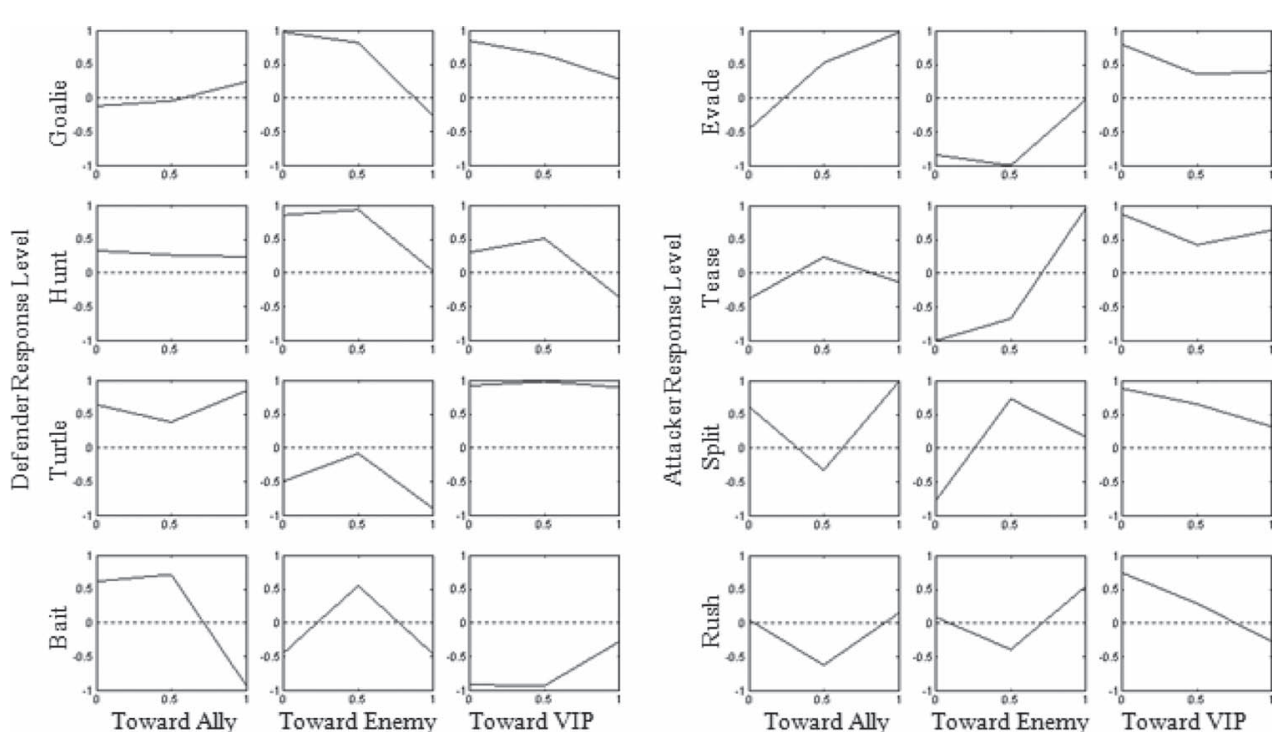

(a)
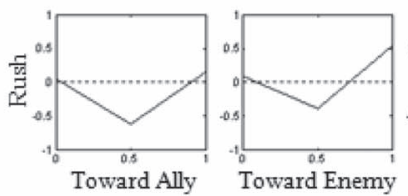

(b)

Fig. 5. (a) Typical defender-evolved genomes. For Goalies, either high ally or center attraction is needed, in addition to enemy attraction for pursuit when nearby. For Hunters, High pursuit is the only consistent requirement. For Turtles, high VIP attraction is observed. For Baits, high VIP repulsion is seen. (b) Typical attacker-evolved genomes. For Evaders, all that is need is enemy repulsion. For Teasers, a springlike or pulsing behavior emerges. For Split, a pulsing behavior occurs among attackers, leading to a controlled dispersal and looser formation. For Rush, the dominant effect is center attraction.

named behavior reflects the agents' ability to counteract the opponent team evolved against, not necessarily the genome's ability to address all other opponent types.

The qualitative observations neither represent all possible variants of attacker and defender behaviors nor demonstrate exact counters between them. The primary rules in Tables I and II reflect the dominant values in the final evolved genomes, but hybrids of Split and Rush, Goalie-Hunt, and others exist and complicate classification. Not all sensors are vital to the observed behavior: for example, Hunting is primarily charac- terized by enemy pursuit. However, Hunter reactions to other allies varied between cycles, with some ignoring allies and others actively repelling. Similarly, not all counters completely defeat a given behavior. There were instances of countered Goalie behaviors being addressed by a new adapted Goalie with only slight variations in ally and enemy responses.

Representative final genomes are shown in Fig. 5. However, these examples are misleading as not all observed behaviors emerged from similar genomes. Many rules displayed disjunctive reasoning; for example, some evolved Goalies 
developed their center-clustering behaviors via strong attraction toward the VIP. Other Goalies, however, ignored the VIP and instead developed strong attractions to each other, thereby swarming the VIP indirectly. The resulting effect is similar, but the genomes emphasize different traits. An unbiased clustering of the entire genomes would overlook the phenotype of the emergent Goalie behavior and group these types separately.

Nonetheless, there is a clear order to the changes of each team's behavioral dynamics resulting from the evolutionary process. For this scenario, teasing attackers are consistently met with Turtling defenders and then counterevolved by sacrificial attackers. These behaviors outline a rulebook of counteracting actions that a swarm can take per the inversion's evolutionary algorithm.

\section{CONCLUSiOn}

WTA solutions often provide optimum target assignments in situations benefitting from global information or direct communication. However, situations involving large multiagent interactions obscure direct analytic inspection of the system. Inverting swarm dynamics via an evolutionary algorithm has been shown to efficiently produce a range of behaviors and counterbehaviors. However, there remains a question as to whether the behavioral responses determined here are optimal tactics. Clearly, the Baiting defenders evolved for Teasing attackers demonstrate a risky seemingly counterproductive response, with defenders abandoning the VIP in order to draw susceptible attackers away. In these solutions, the impartial evolutionary algorithm allows exploitation of the minute and seemingly trivial aspects of a population's genome.

The roughness of the fitness landscape and the genome's performance susceptibility to initial conditions poses a difficult challenge for evolutionary and optimization techniques. The protracted nature of the simulation means that small changes in agent controllers propagate over time into large changes in overall behavior. This scenario poses some interesting results on the issue of autonomous agent tactics. The occasional high performer for a specific initial condition is an impediment to the optimizer. However, these results are still useful for the tactician, as they indicate the forcing conditions necessary to achieve a risky but particularly effective outcome. Inversion of both swarms' dynamics produced unexpected results that formed a useful playbook of operating behaviors for effective team tactics.

\section{ACKNOWLEDGMENT}

This text is derived from Mr. Yu's M.S. thesis.

\section{REFERENCES}

[1] P. Arabshahi, A. Gray, I. Kassabalidis, A. Das, S. Narayanan, M. El-Sharkawi, and R. J. Marks, II, "Adaptive routing in wireless communication networks using swarm intelligence," in Proc. 19th AIAA Int. Commun. Satell. Syst. Conf., Toulouse, France, Apr. 17-20, 2001.

[2] R. Beckers, O. E. Holland, and J. L. Deneubourg, "From local actions to global tasks: Stigmergy and collective robotics," in Proc. Artif. Life IV, 1994, pp. 181-189.

[3] B. E. Bishop, "On the use of redundant manipulator techniques for control of platoons of cooperating robotic vehicles," IEEE Trans. Syst., Man, Cybern. A, Syst., Humans, vol. 33, no. 5, pp. 608-615, Sep. 2003.
[4] E. Bonabeau and C. Meyer, "Swarm intelligence, a whole new way to think about business," Harvard Bus. Rev., vol. 79, no. 5, pp. 106-114, May 2001.

[5] A. K. Das, R. J. Marks, M. A. El-Sharkawi, P. Arabshahi, and A. Gray, "The minimum power broadcast problem in wireless networks: An ant colony system approach," in Proc. IEEE CAS Workshop Wireless Commun. Netw., Pasadena, CA, Sep. 5-6, 2002, pp. 1-4.

[6] P. Dasgupta, "A multiagent swarming system for distributed automatic target recognition using unmanned aerial vehicles," IEEE Trans. Syst., Man, Cybern. A, Syst., Humans, vol. 38, no. 3, pp. 549-563, May 2008.

[7] W. Ewert, D. Jepson, R. J. Marks, II, B. B. Thompson, and A. Yu, "Evolutionary inversion of swarm emergence using disjunctive combs control," Baylor Univ., Waco, TX, Lab Rep. 120601. [Online]. Available: http://www.robertmarks.org/REPRINTS/short/DisjunctiveSwarm.pdf

[8] G. Di Caro and M. Dorigo, "AntNet: Distributed stigmergetic control for communications networks," J. Artif. Intell. Res., vol. 9, no. 1, pp. 317$365,1998$.

[9] M. Dorigo, V. Maniezzo, and A. Colorni, "Ant system: Optimization by a colony of cooperating agents," IEEE Trans. Syst., Man, Cybern. B, Cybern., vol. 26, no. 1, pp. 29-41, Feb. 1996.

[10] J. Golbeck, "Evolving optimal parameters for swarm control," in Proc. NASA-DOD Conf. Evolvable Hardware, 2002, pp. 152-153.

[11] I. A. Gravagne and R. J. Marks, II, "Emergent behaviors of protector, refugee and aggressor swarm," IEEE Trans. Syst., Man, Cybern. B, Cybern., vol. 37, no. 2, pp. 471-476, Apr. 2007.

[12] J. J. Grefenstette, C. L. Ramsey, and A. C. Scchultz, "Learning sequential decision rules using simulation models and competititon," Mach. Learn., vol. 5, no. 4, pp. 335-381, 1990.

[13] P. M. Kanade and L. O. Hall, "Fuzzy ants and clustering," IEEE Trans. Syst., Man, Cybern. A, Syst., Humans, vol. 37, no. 5, pp. 758-769, Sep. 2007.

[14] I. Kassabalidis, M. El-Sharkawi, R. J. Marks, II, P. Arabshahi, and A. Gray, "Adaptive-SDR: adaptive swarm-based distributed routing," in Proc. Int. Joint Conf. Neural Netw., May 12-17, 2002, pp. 351-354.

[15] A. J. Lotka, Elements of Physical Biology. Baltimore, MD: Williams \& Wilkins, 1925.

[16] A. S. Manne, "A target assignment problem," Oper. Res., vol. 6, no. 3, pp. 346-351, May/Jun. 1958.

[17] H. Okada and T. Takagi, "Evaluation of multiobjective genetic algorithm for RoboCupSoccer team evolution," in Proc. SICE Annu. Conf., Aug. 20-22, 2008, pp. 151-154.

[18] R. D. Reed and R. J. Marks, II, "An evolutionary algorithm for function inversion and boundary marking," in Proc. IEEE Int. Conf. Evol. Comput., Nov. 26-30, 1995, pp. 794-797.

[19] C. Reynolds, "Evolution of corridor following behavior in a noisy world," in Proc. 3rd Int. Conf. Simul. Adapt. Behav., 1994, pp. 402-410.

[20] K. M. Sim and W. H. Sun, "Ant colony optimization for routing and loadbalancing: survey and new directions," IEEE Trans. Syst., Man, Cybern. A, Syst., Humans, vol. 33, no. 5, pp. 560-572, Sep. 2003.

[21] P. B. Sujit, A. Sinha, and D. Ghose, "Multiple UAV task allocation using negotiation," in Proc. 5th Int. Joint Conf. Auton. Agents Multi-Agent Syst., Hakodate, Japan, May 8-12, 2006, pp. 147-148.

[22] V. Volterra, "Variazioni e fluttuazioni del numero d'individui in specie animali conviventi," Mem. R. Accad. Naz. Dei Lincei, Ser. VI, vol. 2, pp. 31-113, 1926.

[23] E. Wachholder, "A neural network-based optimization algorithm for the static weapon-target assignment problem," Inf. J. Comput., vol. 1, no. 4, pp. 232-246, 1989.

[24] A. R. Yu, "Optimizing multi-agent dynamics for underwater tactical applications," M.S. thesis, Dept. Elect. Comput. Eng., Baylor Univ., Waco, TX, 2011.

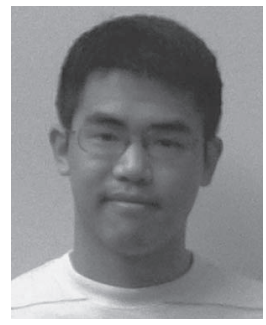

Albert R. Yu received the B.S. degree in bioengineering from Rice University, Houston, TX, in 2007, and the M.S. degree in electrical and computer engineering from Baylor University, Waco, TX, in 2011. He is currently working toward the Ph.D. degree with the University of Washington, Seattle.

His research interests include multiagent system modeling and multiobjective optimization. 


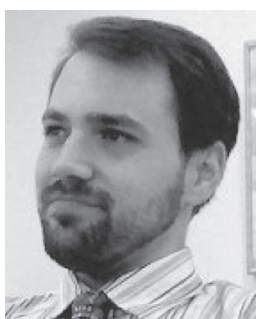

Benjamin B. Thompson received the B.S. degree from Baylor University, Waco, TX, in 1996, and the M.S. and Ph.D. degrees in electrical engineering from the University of Washington, Seattle, in 2002 and 2004, respectively.

$\mathrm{He}$ is currently a Research Engineer and Head of the Tactical Processing Department, Undersea Weapons Office, Applied Research Laboratory, Pennsylvania State University, University Park. His research interests include swarm intelligence, autonomous undersea vehicles, sonar signal processing, multiagent systems, intelligent control systems, and machine learning.

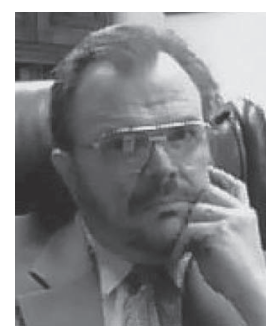

Robert J. Marks, II (S'71-M'72-SM' 83-F'94) is a Distinguished Professor of electrical and computer engineering with the Department of Electrical and Computer Engineering, Baylor University, Waco, TX. His consulting activities include Microsoft Corporation, Pacific Gas \& Electric, and Boeing Computer Services. He served for 17 years as Faculty Advisor to the University of Washington's chapter of Campus Crusade for Christ. His research has been funded by organizations such as the National Science Foundation, General Electric, Southern California Edison, EPRI, the Air Force Office of Scientific Research, the Office of Naval Research, the Whitaker Foundation, Boeing Defense, the National Institutes of Health, The Jet Propulsion Lab, Army Research Office, and NASA.

Prof. Marks is a Fellow of The Optical Society of America and an inductee into the Texas Tech Electrical Engineering Academy. He was the recipient the NASA Tech Brief Award and the Best Paper Award from the American Brachytherapy Society for prostate cancer research, the IEEE Outstanding Branch Councilor Award, The IEEE Centennial Medal, the IEEE Neural Networks Society Meritorious Service Award, the IEEE Circuits and Systems Society Golden Jubilee Award, the IEEE Dallas Section Volunteer of the Year Award for 2007 from the IEEE CIS Chapter, and Distinguished Young Alumnus of Rose-Hulman Institute of Technology. In 2007, he was awarded the Banned Item of the Year from the Discovery Institute. 